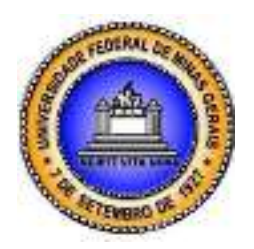

\title{
ANÁlise do PROCESSO DE TRABALHO NA ESTRATÉgia SAÚde DA FAMÍLIA EM RELAÇÃO À OPERACIONALIZAÇÃO DOS PRINCÍPIOS BÁSICOS DO SUS
}

\author{
ANALYSIS OF WORK PROCESS IN THE FAMILY HEALTH STRATEGY IN \\ RELATION TO THE OPERATIONALIZATION OF THE BASIC PRINCIPLES OF \\ SUS
}

\section{ANÁLISIS DEL PROCESO DE TRABAJO EN ESTRATEGIA DE SALUD FAMILIA EN RELACIÓN A LA FUNCIONAMIENTO DE LOS PRINCIPIOS FUNDAMENTALES DEL SUS}

\author{
Michelle Aparecida Vieira \\ Universidade Federal de Viçosa \\ michellevieiracco@gmail.com
}

\author{
Submetido em: 05/04/2014 \\ Correções obrigatória: 06/11/2014 \\ Artigo aceito em: 20/02/2015
}

\section{Marco Aurélio Marques Ferreira \\ Universidade Federal de Viçosa \\ marcoaurelio@ufv.br}

\begin{abstract}
Resumo: A Estratégia Saúde da Família é prioridade do governo federal no âmbito das reformas do serviço público de saúde devido a seu caráter inovador em trabalhar a saúde no país. Nessa vertente, o papel desenvolvido pelos profissionais de saúde é crucial no novo modelo assistencial proposto pelo Ministério da Saúde, bem como a conjuntura em que o programa atua. Sendo assim, buscou-se com o estudo investigar as condições de trabalho dos profissionais da equipe de saúde da família e de que maneira a realidade vivenciada pelos mesmos influencia na operacionalização dos princípios básicos do SUS. O processo de trabalho foi avaliado por meio de constructos, que apresentaram valores referentes aos principais aspectos envolvidos no funcionamento do programa. Os resultados encontrados revelaram que as condições estruturais das Unidades, assim como os equipamentos e instrumentos necessários para o desenvolvimento das práticas de saúde são insatisfatórios, o que compromete o atendimento realizado pela equipe. Contudo, conclui-se que a aceitação da ESF pela sociedade favoreceu o cumprimento de um dos objetivos do programa que é reafirmar os princípios do SUS, principalmente quando estende os serviços públicos de saúde à camada mais pobre da população.

PALAVRAS-CHAVE: estratégia saúde da família; processo de trabalho; princípios do SUS.
\end{abstract}

\begin{abstract}
The Family Health Strategy is a priority of the federal government within the public health service reforms due to its innovative character working health in the country. In this strand, the role played by health professionals is crucial in the new care model proposed by the Ministry of Health, as well as conjuncture wherein the program operates. Therefore, we sought with the study investigate the working conditions of health professionals from the family and how the reality experienced by the same influence in the operationalization of the basic principles of the SUS. The work process was evaluated by means of constructs that demonstrate values related the main aspects involved in the operation of program. The results found showed that the structural conditions of the Units, as well as the equipment and instruments necessary for the execution of health practices are unsatisfying, which compromise the care realized by staff. However, concluded that the acceptance of the ESF by society favor the fulfillment of one of the goals of the program that is reaffirm the principles of the SUS, mainly when extend the public health services to the poorest strata of the population.
\end{abstract}

KEYWORDS: the family health strategy; work process; principles of the SUS.

Resumen: La Estrategia de Salud de la Familia es una prioridad del gobierno federal dentro de las reformas de los servicios de salud pública debido a su carácter innovador en trabajo la salud en el país. En este aspecto, el papel desempeñan por los profesionales de la salud es fundamental en el nuevo modelo de atención propuesto por el Ministerio de Salud así como la coyuntura en que opera el programa. Por lo tanto, buscamos con el estudio 

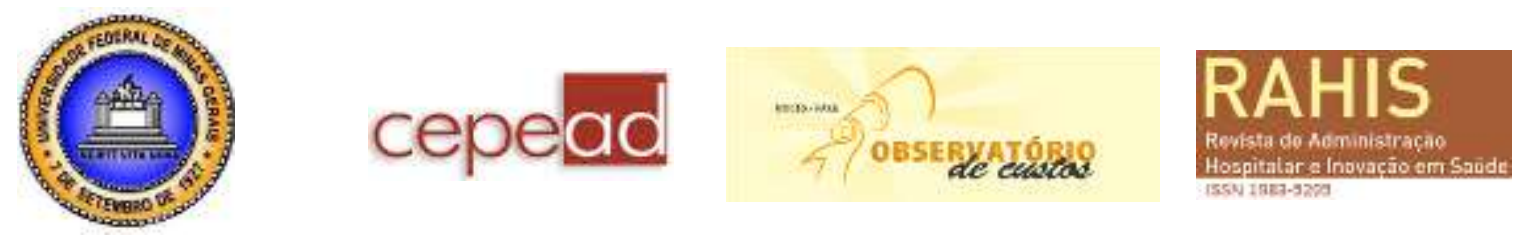

investigar las condiciones de trabajo de los profesionales de salud de la familia y cómo la realidad vivida por las mismas influencias en la operacionalización de los principios básicos de la SUS. El proceso de trabajo se evaluó por medio de constructos que demostraron valores para los principales aspectos que intervienen en la operación del programa. Los resultados mostraron que las condiciones estructurales de las unidades, así como los equipos y instrumentos necesarios para el desarrollo de las prácticas de salud son insatisfactorios, lo que compromete la tratamiento brindada por el equipo. No obstante, concluye que la aceptación del ESF por la sociedad promueve el cumplimiento de uno de los objetivos del programa que es reafirmar los principios del SUS, sobre todo cuando extiende los servicios de salud pública a los estratos más pobres de la población.

PALABRAS -CLAVE: the family health strategy; work process; principles of the SUS.

\section{INTRODUÇÃO}

Um dos desafios do Ministério da Saúde tem sido adequar as práticas de saúde às constantes transformações epidemiológicas decorrentes do crescimento populacional, fator este que influencia diretamente no processo saúde/doença. Na perspectiva de atender as novas necessidades de saúde, foi proposto pelo governo federal a criação de uma política pública capaz de reorientar as ações de saúde, mudando significativamente a maneira de trabalhar a saúde no Brasil.

Foi assim que em 1994 nasceu o Programa Saúde da Família (PSF), como parte do processo de reforma do setor de saúde, desde a promulgação da Constituição de 1988 que introduziu a saúde como um direito de todo cidadão. Esse novo modelo assistencial rompe com o paradigma do sistema centrado na medicina curativa e adota como mecanismo de intervenção as ações de promoção, prevenção e recuperação da saúde.

Tem como principal característica a formação de uma equipe multiprofissional. $\mathrm{O}$ trabalho desenvolvido pelas equipes é o elemento-chave na tentativa de reorientar as ações de saúde praticadas, isso porque introduz uma nova visão no processo de intervenção em saúde, na medida em que não espera a população chegar para ser atendida, mas age de forma preventiva sobre ela (ROSA; LABATE, 2005).

Devido a suas características o PSF foge a concepção de programa por ser estratégia prioritária do Ministério da Saúde para organizar a atenção básica, através da promoção e reorientação das práticas e ações de saúde, de forma integral e contínua (MONTEIRO; FIGUEIREDO; MACHADO, 2008). Posto isso, o programa passou a ser conhecido como Estratégia Saúde da Família- ESF. Esta estratégia do governo propõe uma nova dinâmica na forma de organização dos serviços de saúde, uma vez que a mudança do enfoque de assistência à doença para a promoção da saúde exige atuação articulada com outros setores da sociedade, tendo como resultado positivo a qualidade nos serviços prestados à população (CAMELO; ANGERAMI, 2008).

$\mathrm{Na}$ literatura, existem estudos que descrevem experiências envolvendo a Estratégia Saúde da Família. Viana Dal Poz (1998) aborda a implantação do Programa Saúde da Família como parte do processo de reforma do sistema de saúde no Brasil. Maia e Santos (2011) discutem sobre a qualidade de vida na ESF. Araújo e Rocha (2007) demonstram a importância do trabalho em equipe na consolidação da Estratégia Saúde da Família. Nesse estudo, o objetivo foi analisar a percepção dos profissionais em relação às condições de trabalho as quais estão submetidos, tendo em vista que o trabalho desenvolvido por eles assume papel determinante no novo modelo assistencial proposto pelo Ministério da Saúde. 

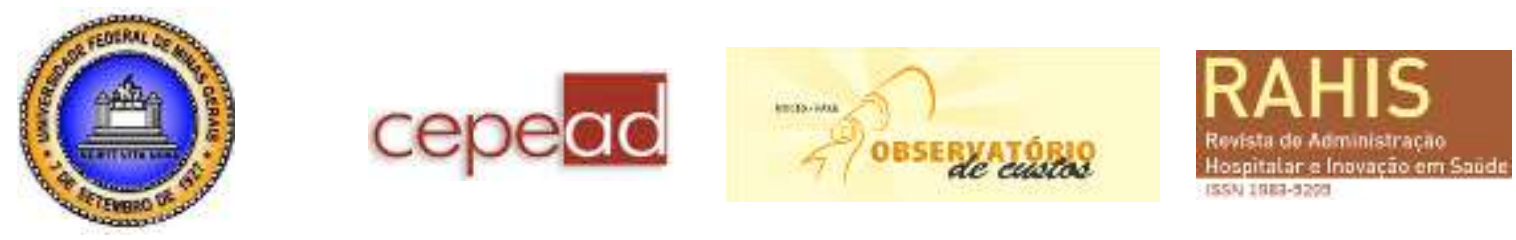

Partindo do pressuposto de que a ESF está inserida na Atenção Básica e que assume "papel potencial de eixo norteador da organização dos serviços de saúde municipais" (CAMELO; ANGERAMI, 2008, p. 46) ressalta-se a relevância de verificar a existência dos princípios básicos do SUS- universalização, descentralização, integralidade, equidade, universalidade e participação social nas ações desenvolvidas pelos profissionais, visto que o programa incorpora e reafirma esses princípios.

Pretende-se com este estudo contribuir no aperfeiçoamento da gestão dos serviços públicos de saúde, pois uma vez identificadas as dificuldades existentes no ambiente de trabalho dos profissionais é possível canalizar investimentos nas áreas de maior carência, seja de recursos físicos ou financeiros, a fim de adequar a assistência prestada aos princípios norteadores da ESF, tendo como resultado principal a qualidade dos serviços de saúde.

\section{FUNDAMENTAÇÃO TEÓRICA}

\section{Reorientação do modelo assistencial- SUS}

A promulgação da Constituição Federal em 1988 estabeleceu o acesso à saúde um direito de todo cidadão, cabendo ao Estado o dever de proporcionar condições necessárias para que esse direito seja usufruído, mediante a criação de "políticas sociais e econômicas que visem à redução do risco de doença e de outros agravos e ao acesso universal e igualitário às ações e serviços para sua promoção, proteção e recuperação" (BRASIL, 1988, Art. 196).

Para tanto, foi criado o Sistema Único de Saúde (SUS), um sistema público responsável por oferecer ações e serviços de saúde de forma igualitária e gratuita a toda população. Trata-se, portanto de uma nova formulação política e organizacional para o redirecionamento dos serviços e ações de saúde. Este sistema é regulamentado pelas Leis Orgânicas da Saúde (Leis $n^{\circ}$ 8.080/90 e $n^{\circ}$ 8.142/90), que dispõe sobre as ações e serviços de saúde, e os princípios e diretrizes que o norteiam.

Por meio da implantação do SUS, a atenção à saúde no Brasil tem alcançado novos contornos, atribuindo novas responsabilidades e competências a cada nível do governo, principalmente aos municípios que se tornaram responsáveis pela gestão do sistema local de saúde (MARTINS; GARCIA; PASSOS, 2008).

A concepção do SUS é norteada por algumas doutrinas, quais sejam: a universalidade, a equidade e a integralidade das ações de saúde. A universalidade implica o direito ao atendimento; a equidade parte da premissa de que todo cidadão é igual perante o SUS e será atendido conforme as suas necessidades; a integralidade implica que todas as ações de promoção, proteção e de recuperação da saúde formem um todo indivisível, que não pode ser compartimentalizado (OLIVEIRA et al, 2008).

Esse novo modelo assistencial definiu a descentralização municipalizante e propôs uma nova forma de organizar os serviços de saúde perante a lógica da integralidade, regionalização e hierarquização, definindo dessa forma a porta de entrada a Atenção Básica (VIANA; DAL POZ, 1998).

A implementação do sistema propiciou uma evolução no campo das políticas públicas de saúde, visto que através do princípio da descentralização, todos os entes governamentais são responsáveis de prover mecanismos que garantem à população o acesso aos serviços de 

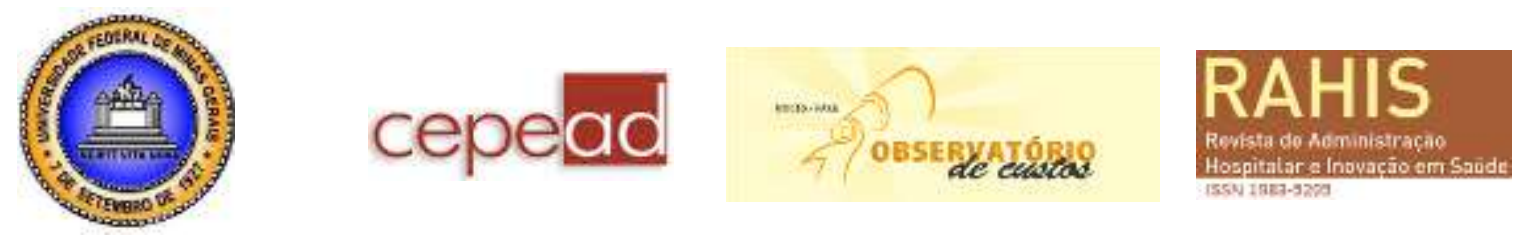

saúde. Trata-se, portanto de uma estrutura organizacional, instituída na área da saúde, com a finalidade de atender à demanda da clientela, a partir de uma mudança nas formas de direcionar e conceber a assistência à saúde no país (OLIVEIRA et al, 2008).

Ainda segundo Oliveira et al (2008), o SUS tem como objetivos finais:

\begin{abstract}
Prestar assistência à população a partir do modelo de promoção da saúde, que implica ações buscando eliminar ou controlar as causas das doenças e agravos, ou seja, determinando ou condicionando o aparecimento de doenças; proteger a saúde da população, que consiste em ações especificas para prevenir riscos e exposições às doenças e agravos à saúde, ou seja, manter o seu estado de saúde; e desenvolver ações de recuperação da saúde de forma a evitar mortes e sequelas em pessoas já acometidas por processos mórbidos (OLIVEIRA et al, 2008, p. 198).
\end{abstract}

O SUS tem como propósito "tornar-se um importante mecanismo de promoção da equidade no atendimento das necessidades de saúde da população, ofertando serviços com qualidade adequados às necessidades, independente do poder aquisitivo do cidadão" (BRASIL, 2013b).

Grandes foram os avanços do Ministério da Saúde no campo da saúde, através de suas ações e programas que revolucionaram as práticas de saúde existentes. Como exemplo destaca-se o Programa Saúde da Família (PSF), criado em 1994 com o principal propósito de "reorganizar a prática da atenção à saúde em novas bases e substituir o modelo tradicional, que se configurava" (COSTA; TRINDADE; PEREIRA, 2010, p.1).

Rosa e Labate (2005) afirmam que a busca por novos modelos de assistência é decorrente de um momento histórico/social em que o modelo tecnicista/hospitalocêntrico não atende mais às mudanças do mundo contemporâneo e, consequentemente, às necessidades de saúde das pessoas. Nesse contexto, o PSF surge para incorporar um conjunto de questões relativas às bases conceituais e operacionais do que se tem denominado "Atenção Básica à Saúde" que está inserida num contexto de descentralização e controle social da gestão dos programas de saúde do SUS.

\title{
Estratégia Saúde da Família e o papel da equipe multiprofissionalizante
}

A origem do Programa Saúde da Família (PSF) remete a criação do Programa de Agentes Comunitários da Saúde (PACS) em 1991, como parte do processo de reforma do sistema de saúde pública vigente no Brasil. O PACS foi formulado com o intuito de reduzir os índices de mortalidade infantil e materna nas regiões Norte e Nordeste do país, através da expansão da cobertura dos serviços de saúde para as áreas mais carentes. (VIANA; DAL PÓZ, 1998).

O êxito alcançado com a implementação do PACS no Ceará fez com que o Ministério da Saúde (MS) percebesse a importância dos agentes nos serviços básicos de saúde no município e começasse a enfocar a família como unidade de ação programática de saúde, não enfocando somente o indivíduo, mas introduzindo a noção de área por cobertura (família) (BRASIL, 1997).

A partir de 1994, o PSF foi se expandindo pelo resto do país tornando-se uma estratégia para a reorganização e fortalecimento da atenção básica como o primeiro nível de 

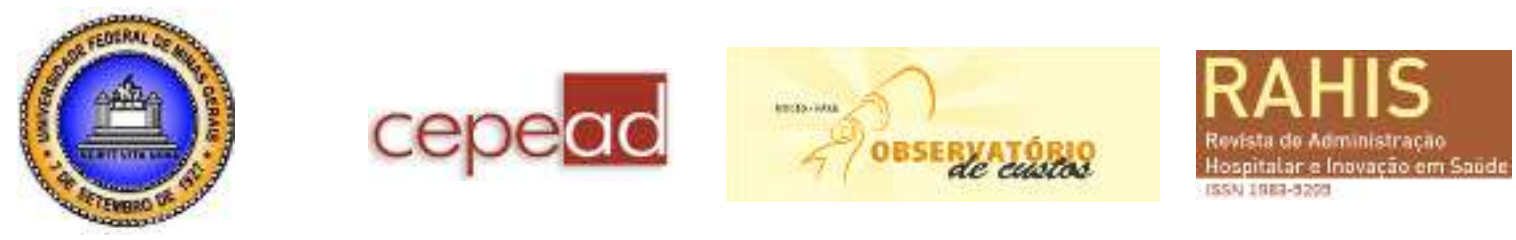

atenção à saúde no Sistema Único de Saúde (SUS), através da ampliação do acesso, qualificação e reorientação das práticas de saúde.

Embora o PSF seja classificado como um programa, por suas características, foge a concepção usual dos demais programas criados pelo governo, uma vez que não se trata de uma intervenção vertical e paralela as atividades de saúde. Tal programa "caracteriza-se como uma estratégia que possibilita a integração e promove a organização das atividades em um território definido, com o propósito de propiciar o enfrentamento e resolução dos problemas identificados" (BRASIL, 1997).

Em 2006 o PSF deixou de ser considerado programa e passou a ser uma estratégia na atenção básica em saúde, em razão de possuir tempo determinado e a estratégia ser permanente e contínua. Sendo assim, passou a ser denominado de Estratégia Saúde da Família - ESF (DALPIAZ; STEDILE, 2011).

De acordo com Camelo e Angerami (2008, p.45), a Estratégia de Saúde da Família (ESF) está inserida em "um contexto de decisão política e institucional de fortalecimento da atenção básica no âmbito do SUS". Tem como principal finalidade a geração e análise de dados que possam produzir informações sobre a necessidade de saúde, organizando o processo de equipe e intervindo nas necessidades de saúde das famílias.

Para o Ministério da Saúde, a "Saúde da Família é entendida como uma estratégia de reorientação do modelo assistencial, operacionalizada mediante a implantação de equipes multiprofissionais em unidades básicas de saúde" (BRASIL, 2013a). Essas equipes atuam nas unidades básicas de saúde, nas residências e na mobilização da comunidade, promovendo o acesso aos serviços de saúde a todos os segmentos da sociedade.

Os profissionais das equipes de saúde serão responsáveis por sua população adstrita, devendo residir no município onde atuam, trabalhando em regime de dedicação integral. A fim de garantir a vinculação com as famílias sob sua responsabilidade, os Agentes Comunitários de Saúde (ACS) devem, igualmente, residir nas suas respectivas áreas de atuação (BRASIL, 1997).

A base de atuação das equipes é a Unidade Básica de Saúde (UBS), no entanto suas atividades se expandem para o trabalho em campo que configura as visitas domiciliares, com a finalidade de acompanhar a situação das famílias cobertas pelo sistema. Nesse sentido, o Ministério da Saúde preconiza que as unidades de Saúde da Família sejam implantadas em conformidade com as diretrizes que orientam o programa, de tal modo que se adeque às realidades regionais, municipais e locais em que estão inseridas.

Camelo e Angerami (2008) mencionam que por se tratar de um novo modelo de assistência que prioriza o trabalho em equipe, é fundamental destacar a importância da formação de Recursos Humanos voltadas às necessidades dos profissionais, e concluem:

Este modelo de atenção à saúde, conforme proposto, leva-nos a pensar que, como se trata de uma estratégia do Ministério da Saúde (MS) cujo objetivo é reordenar a assistência através de um trabalho essencialmente em equipe, no qual são delegadas aos profissionais inúmeras atribuições com alto grau de exigências e responsabilidades, os membros dessas equipes necessitam de conhecimentos e habilidades específicas para assistir a comunidade extramuros, além de criatividade e adequado suporte técnico-científico (CAMELO; ANGERAMI, 2008, p. 46). 

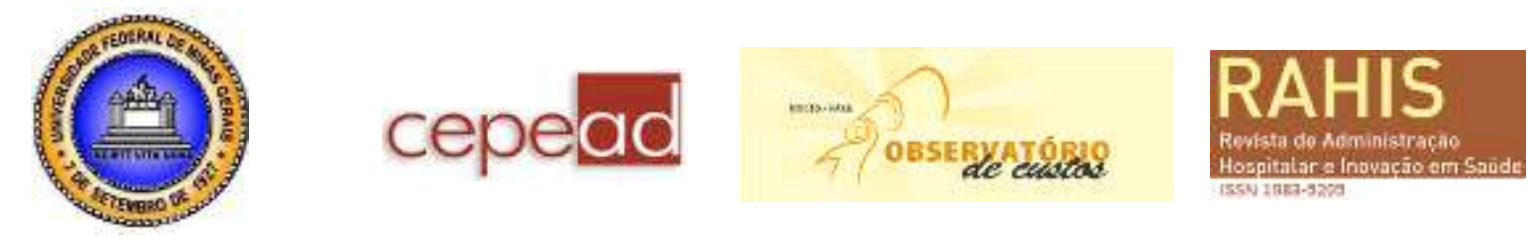

Nota-se que as ações desenvolvidas pela equipe de saúde envolvem conhecimentos e habilidades específicas, o que demanda adequada preparação dos profissionais envolvidos na realização das atividades.

\section{PROCEDIMENTOS E MÉTODOS}

\section{Caracterização do município estudado:}

O estudo foi realizado no município de Ubá, em Minas Gerais, que conta com a atuação de 18 (dezoito) equipes de saúde da família, responsáveis por cobrir uma área de aproximadamente 62.100, representando cerca de 60,42\% da população (BRASIL, 2013c). Do total de equipes atuantes no município, a pesquisa contou com a participação de 11(onze), uma vez que estas se mostraram dispostas a contribuírem na realização do estudo.

A escolha do município justifica-se pelo fato de ser destaque na região da Zona da Mata, sendo o segundo mais desenvolvido economicamente nesta região.

\section{Métodos analíticos e de coleta:}

Inicialmente foi feita uma consulta nos documentos oficiais de domínio público, que abordam as particularidades do programa, com o intuito de investigar quais são as recomendações propostas pelo governo em relação ao programa e contrapor à realidade encontrada nas unidades de saúde do munícipio analisado.

No desenvolvimento da pesquisa, foi utilizado como instrumento de coleta de dados questionários estruturados, os quais foram aplicados aos enfermeiros e agentes de saúde de cada unidade. O questionário é composto de duas etapas: a primeira, destinada apenas aos enfermeiros, buscou conhecer o perfil dos profissionais que compõe a ESF.

A segunda parte compreende um bloco de opinião destinado a ambos profissionais. As questões dos blocos de opinião foram elaboradas de maneira a facilitar a formação dos constructos. Para isso, a ordenação das questões foi feita com base na "técnica do funil", segundo a qual cada questão deve relacionar-se à questão anterior a fim de apresentar maior especificidade (GIL,2006).

Através dos dados obtidos com os questionários, foi feita a Análise Descritiva dos Dados, a qual, conforme Bruni (2007) tem como principal função resumir os dados e informações coletadas, apresentando-as de maneira a facilitar as principais características do objeto de estudo, neste caso a Estratégia Saúde da Família.

Para analisar a situação de trabalho dos profissionais da Estratégia Saúde da Família, optou-se pela elaboração de constructos que agrupassem as informações coletadas de acordo com suas características semelhantes.

Para isso, fez-se necessário verificar a correlação entre as variáveis estudadas. A correlação indica o quanto duas ou mais variáveis estão relacionadas, podendo essa relação ser linear positiva ou negativa. Duas variáveis são ditas correlacionadas se mudanças em uma são associadas com mudanças na outra, sejam essas mudanças na mesma direção ou direções opostas (HAIR; ANDERSON; TATHAM, 2009). 


\section{Elaboração dos constructos e construção da escala:}

A elaboração dos constructos foi feita com base na escala Likert, a qual conforme Malhotra (2006) é uma escala de medidas que utiliza cinco ou sete categorias de respostas, variando de "concordo totalmente" a "discordo totalmente", considerando também a opção de indiferente. Nesta escala, os participantes apontam seu grau de concordância ou discordância em relação às afirmativas apresentadas, sendo que uma resposta "concordo totalmente" representa uma afirmação favorável e "discordo totalmente", uma afirmação desfavorável.

Um CONSTRUCTO é uma ideia genérica formada pela combinação de uma série de características semelhantes às quais são as variáveis que coletivamente definem e tornam a mensuração do conceito possível (HAIR; ANDERSON; TATHAM, 2009).

Neste estudo optou-se por utilizar cinco categorias de respostas, por entender que um número muito grande de alternativas poderia prejudicar a escolha, e o número ímpar de alternativas permite a neutralidade do respondente, sendo que estas são suficientes para alcançar os objetivos pretendidos. As categorias estão dispostas de forma contínua e assimétrica, como demonstrada no quadro a seguir.

Quadro 1: Método de elaboração das categorias

\begin{tabular}{|c|c|c|c|c|}
\hline $\mathbf{1}$ & $\mathbf{2}$ & $\mathbf{3}$ & $\mathbf{4}$ & $\mathbf{5}$ \\
\hline $\begin{array}{c}\text { Concordo } \\
\text { totalmente }\end{array}$ & $\begin{array}{c}\text { Concordo } \\
\text { Parcialmente }\end{array}$ & Indiferente & $\begin{array}{c}\text { Discordo } \\
\text { Parcialmente }\end{array}$ & $\begin{array}{c}\text { Discordo } \\
\text { Totalmente }\end{array}$ \\
\hline
\end{tabular}

Fonte: Elaboração dos autores, 2013.

A fim de atribuir valor ao constructo, que é o conjunto de variáveis correlacionadas, foi calculada a média a partir dos resultados obtidos com as respostas, ou seja, foram somados os pontos de 1 a 5 e dividido o valor encontrado por 5, número que representa a quantidade de variáveis. Desse modo, quanto menor o valor do constructo, melhor é a percepção dos profissionais de saúde quanto aos aspectos abordados.

Os constructos foram elaborados a partir da combinação de uma série de características semelhantes, no intuito de apresentar conjuntamente aquelas que mais se relacionam, conforme representa a Figura 1. 


\section{cepead}
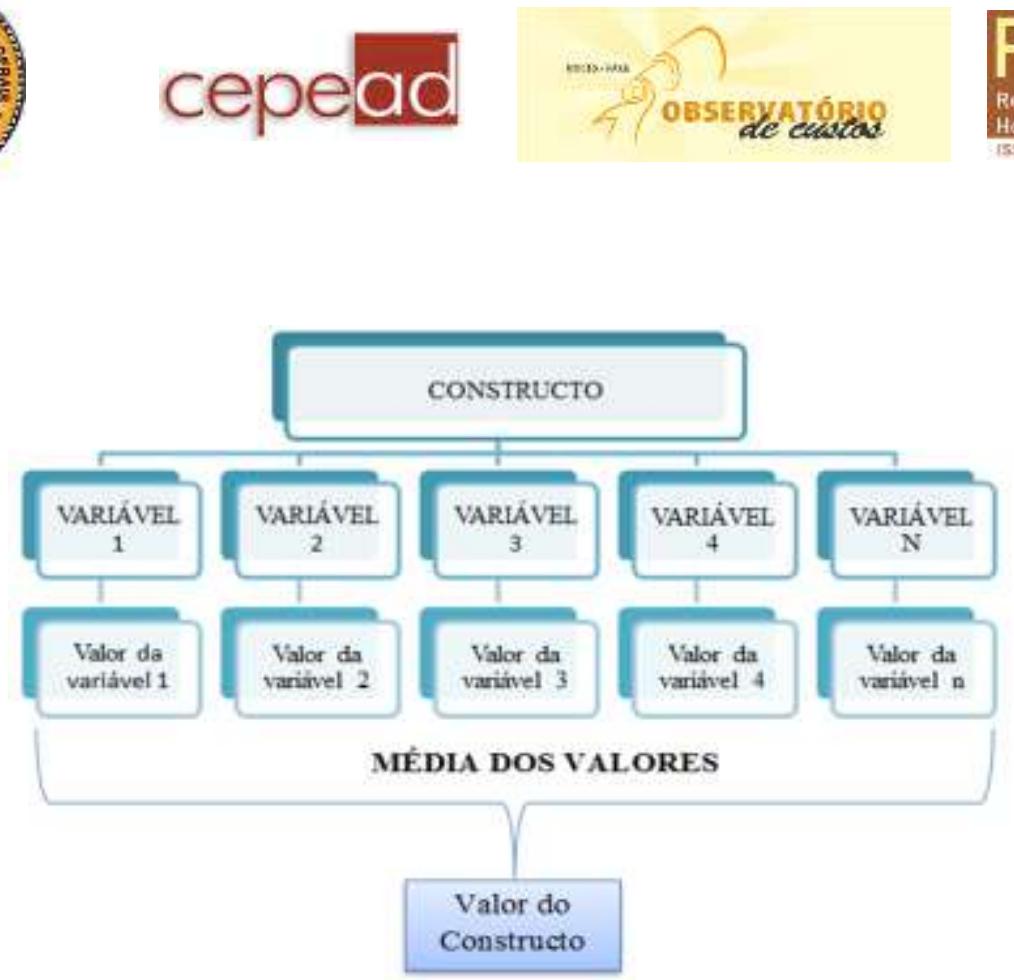

FIGURA 1: Processo de elaboração dos constructos

Fonte: Elaboração dos autores, 2013.

\section{Validação dos constructos: Alfa de Cronbach}

Para validar os constructos, foi utilizado como teste de validação o Alfa de Cronbach, o qual segundo Hair, Anderson e Tatham (2009), determina se três ou mais itens combinados em um único índice captam, de maneira coerente, o constructo.

Trata-se de um coeficiente de confiabilidade muito utilizado para verificar se uma escala está de acordo com sua definição conceitual e se atende aos níveis necessários de confiabilidade. É definido pela fórmula:

$$
\alpha=\frac{n\left(\frac{\operatorname{cov}}{v a r}\right)}{1+(n-1)\left(\frac{\operatorname{cov}}{v a r}\right)}
$$

Em que:

Cov = Média de covariância entre os pares de itens;

Var = Média de variância entre os pares de itens e

$n=$ Número de itens (variáveis).

O Alfa de Cronbach pode assumir vários valores de corte para validar os constructos, podendo variar de 0 a 1 . Caso o resultado seja um número inferior a zero, ou seja, negativo, significa que o constructo violou o pressuposto fundamental da técnica, que é a correlação positiva dos itens. Na literatura existem várias sugestões, sendo 0,60 o valor mínimo recomendado e o utilizado neste estudo.

Para Malhotra (2006, p. 278), "ao determinar a validade de um constructo, o pesquisador procura responder a questões teóricas sobre as razões pelas quais a escala funciona e que deduções podemos fazer sobre a teoria subjacente". 


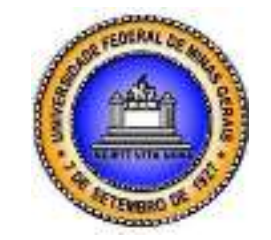

\section{RESULTADOS E DISCUSSÕES}

\section{Cobertura de atendimento e Processo de Trabalho nas equipes de saúde da família}

O município de Ubá conta com a atuação de 18 equipes de saúde da família responsáveis pela cobertura de $60,42 \%$ da população. No estudo, foram selecionadas 11 equipes de saúde, das quais participaram das entrevistas 2 profissionais de cada: um enfermeiro e um agente comunitário.

Os entrevistados foram questionados sobre a preparação inicial para compor a equipe de trabalho. A essa questão, 63,6\% dos entrevistados alegaram que não receberam nenhum tipo de treinamento específico para atuar na Estratégia Saúde da Família, além dos estágios realizados como parte das exigências da formação acadêmica. Segundo o Ministério da Saúde (MS) (BRASIL, 1997), o treinamento inicial é parte de um processo de capacitação na qual são trabalhados aspectos gerais das atividades a serem desenvolvidas pelos profissionais assim como a adaptação do conteúdo programático às necessidades locais. Após o período preparatório, a equipe de saúde deve estar apta a detectar e informar as características de integração do município ao SUS, além de cumprir os princípios operacionais da Estratégia Saúde da Família (ALVES; SILVA, et al. 2010). Observou-se também que em todas as Unidades Básicas, os agentes de saúde realizam as visitas domiciliares em média uma vez ao mês, como proposto pelo Ministério da Saúde. As visitas às residências é um diferencial do programa, uma vez que busca a integração da equipe com a comunidade adstrita, possibilitando a identificação e rápida resolutividade dos problemas existentes. Nos dizeres de Martha et al (p. 161, 2011), " o território, o trabalho em equipe, intersetorialidade e a população adstrita constituem eixos fundamentais da ESF, sendo a visita domiciliar uma de suas principais diretrizes, que objetiva ampliar o acesso aos serviços e criar vínculos com a população".

No que tange a jornada de trabalho, identificou-se que em todas as Unidades, os médicos são os únicos profissionais que cumprem uma carga horária de 30 horas semanais, à medida que os demais membros, perfazem uma carga horária de 40 horas semanais. Sobre essa questão, a Portaria $\mathrm{n}^{\mathrm{o}} 2.027$, de 25 de agosto de 2011, que dispõe sobre a carga horária dos profissionais médicos que compõem as Equipes de Saúde da Família (ESF), determina para a ESF o cumprimento de horário integral, através de uma jornada de trabalho de 40 horas semanais, podendo ser reduzida a 30 horas, para os profissionais médicos, nas Unidades que possuem mais de um médico integrado à equipe, o que não foi constatado nas equipes entrevistadas, que conta com a colaboração de apenas um médico generalista.

\section{Caracterização e qualificação das equipes de saúde}

As equipes de saúde são compostas basicamente por um médico, um enfermeiro, dois técnicos de enfermagem e quatro agentes comunitários, conforme recomendado pelo Ministério da Saúde. Em algumas unidades, a equipe contava também com a colaboração de um dentista e um técnico em higiene bucal, o que complementava as atividades desenvolvidas. 

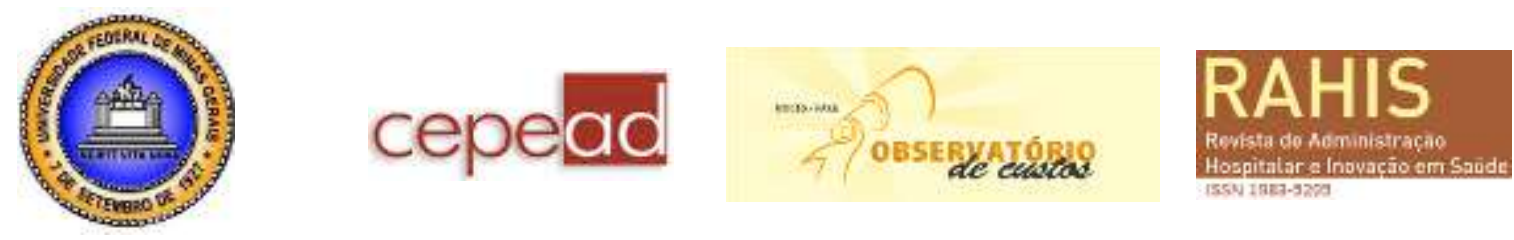

Em relação aos cursos de atualização, 90,9\% dos entrevistados relataram participar de cursos oferecidos pelas Secretarias de Saúde, a fim de inteirar-se sobre as campanhas propostas pelo MS.

No que diz respeito ao monitoramento, $72,7 \%$ dos entrevistados mencionaram ser realizado através do controle da produção, em que deve ser atingido um número mínimo de atendimentos. A partir disso, se alcançado as metas estabelecidas, pode-se inferir que os membros da equipe atenderam de forma satisfatória as expectativas pré-estabelecidas.

\section{Relações de trabalho entre os profissionais da saúde e a comunidade}

A satisfação no ambiente de trabalho constitui um elemento fundamental no contexto em que esta inserida a ESF, uma vez que o vínculo estabelecido entre a equipe de saúde da família e os usuários, decorrente da boa interação entre eles, contribui para o êxito do programa. Sendo assim, constatou-se que apesar de não existir instrumento de motivação profissional, 54,5\% dos respondentes alegaram estar satisfeitos com seu trabalho, o que implica em melhores serviços oferecidos à população.

Outro fator relevante na relação dos profissionais de saúde com a comunidade local diz respeito à humanização no atendimento oferecido nas unidades. Sobre esse aspecto, os resultados apontam que $40 \%$ dos entrevistados entendem que humanização é o acolhimento oferecido aos pacientes no momento das consultas. Segundo Ayres et al (2006, p. 308), acolher significa "receber bem, ouvir a demanda, buscar formas de compreende-la e solidarizar-se com ela."

Em todas as unidades observou-se que os profissionais consideram o atendimento realizado por eles humanizado. A humanização reafirma um dos princípios doutrinários do SUS que é a integralidade no atendimento, no qual o acolhimento deve ser visto, como um dispositivo potente para atender a exigência de acesso, propiciando vínculo entre equipe e população, trabalhador e usuário, além de possibilitar o questionamento do processo de trabalho, desencadeando assim no cuidado integral (SOUZA et al 2008).

\section{Análises dos Constructos}

As informações relacionadas ao processo de trabalho dos profissionais foram apresentadas por meio dos constructos, elaborados com base nas variáveis descritas a seguir e explicadas pelas respectivas questões em que foram fundamentadas. As respostas às questões expostas foram mensuradas com base na escala Likert, variando de 1 a 5 , contendo a opção indiferente.

Registra-se que os resultados encontrados não apresentam heterogeneidade entre a amostra, pois o erro padrão dos constructos foi inferior às médias.

Convém salientar também que todos os constructos apresentaram Alfa de Crombach superior a 0,6, mostrando que são relevantes e que são baseados na percepção dos colaboradores em relação às afirmativas expostas, conforme apresentado na Tabela 1 a seguir: 

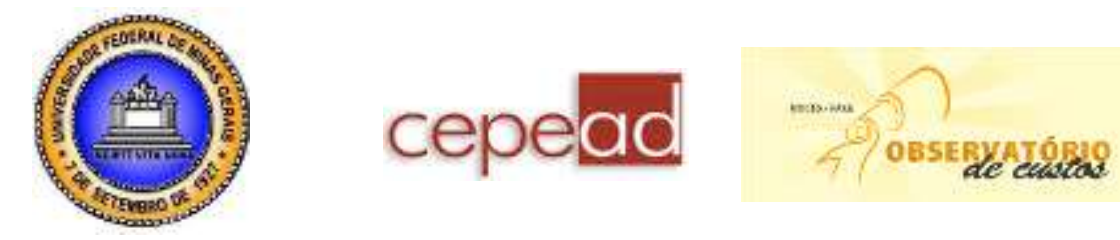

Tabela 1: Valor dos constructos

\begin{tabular}{|c|c|c|c|c|c|}
\hline \multirow{7}{*}{ 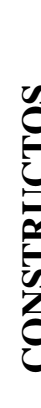 } & & VALOR & $\overline{\text { ALPHA }}$ & $\mathrm{N}^{0}$ ITENS & ERRO PADRÃO \\
\hline & CLIMA ORGANIZACIONAL & 1,9048 & 0,669 & 5 & 0,57138 \\
\hline & INFRAESTRUTURA & 2,869 & 0,723 & 4 & 0,97346 \\
\hline & RECEPTIVIDADE & 1,9881 & 0,615 & 4 & 0,5835 \\
\hline & GESTÃO & 2,4571 & 0,815 & 5 & 0,92118 \\
\hline & CARGA HORÁRIA & 2,2262 & 0,881 & 4 & 1,31554 \\
\hline & SALÁRIOS & 2,8095 & 0,663 & 3 & 1,12828 \\
\hline & RESULTADO & 2,1905 & 0,637 & 3 & 0,76428 \\
\hline
\end{tabular}

Fonte: Elaboração dos autores, 2013.

\section{Clima Organizacional}

Esse constructo foi construído na tentativa de conhecer a relação de trabalho entre os membros da equipe, se existe companheirismo, comunicação, motivação e outros fatores que influenciam a interação entre eles. As variáveis que o compõe estão apresentadas na Tabela 2:

Tabela 2: Constructo Clima Organizacional

\begin{tabular}{|c|c|c|}
\hline Constructo & Variáveis & Questões \\
\hline \multirow{5}{*}{ 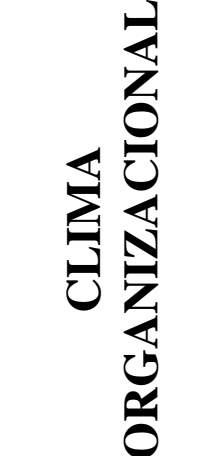 } & Informações Compartilhadas & $\begin{array}{l}\text { Os agentes passam as informações corretamente para os } \\
\text { demais membros da equipe }\end{array}$ \\
\hline & Diálogo entre a equipe & $\begin{array}{l}\text { Existe diálogo entre os enfermeiros, médicos e agentes } \\
\text { comunitários. }\end{array}$ \\
\hline & Participação no Planejamento & $\begin{array}{l}\text { Os médicos participam do planejamento da Unidade } \\
\text { Básica. }\end{array}$ \\
\hline & Orientação & $\begin{array}{l}\text { Os agentes são orientados pelos enfermeiros e técnicos } \\
\text { em enfermagem. }\end{array}$ \\
\hline & Reconhecimento & Você se sente reconhecido pelo trabalho que executa. \\
\hline
\end{tabular}

Fonte: Elaboração dos autores, 2013.

O constructo apresentou valor de 1,90 e aponta a existência de um clima organizacional propício para uma boa convivência no ambiente de trabalho. Sánchez et al. (2006) destacam que ambientes com um bom clima organizacional facilita a realização das atividades de maneira confortável, além de produzir maior satisfação no trabalho e maior potencial dos trabalhadores.

Nesse constructo, a variável melhor conceituada entre os membros da equipe de saúde foi a "orientação", obtendo média de 1,18. Para estes, os enfermeiros cumprem sua função quando orientam os técnicos e agentes nos procedimentos a serem adotados em cada atividade especifica. Esse resultado também é confirmado por meio da variável diálogo entre a equipe, admitindo esta variável a segunda melhor média dentro do constructo, 1,33. Quando existe diálogo entre os membros de um grupo, a interação entre estes possibilita que as informações relevantes sejam repassadas de maneira adequada e tempestiva aos profissionais, de modo que 

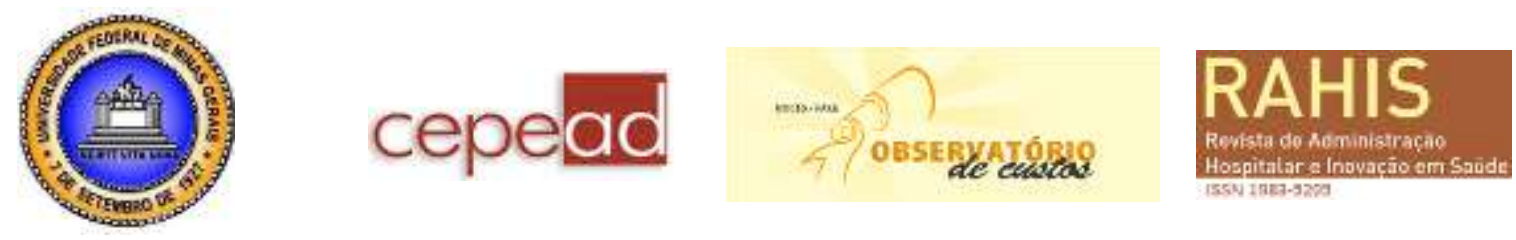

estes conduzam suas atividades de acordo com as necessidades de cada paciente (OLIVEIRA; SPIRI, 2006). Além disso, o diálogo incentiva a comunicação interna no grupo e permite resolver os conflitos e problemas que possam surgir no ambiente de trabalho (CARVALHO; CUNHA, 2006).

Dentre as variáveis desfavoráveis a um bom clima organizacional dentro da Estratégia Saúde da Família, os respondentes apontaram o "reconhecimento" como um dos pontos fracos, obtendo uma média de 2,6. Esse valor mostra que a desmotivação dos profissionais é atribuída a falta de reconhecimento das atividades que executa.

\section{Infraestrutura}

A infraestrutura diz respeito às condições físicas necessárias para a realização das atividades propostas pela ESF. Inclui desde o espaço físico em que é feito os atendimentos até os equipamentos e medicamentos imprescindíveis à realização dos mesmos.

Tabela 3: Constructo Infraestrutura

\begin{tabular}{lll}
\hline Constructo & \multicolumn{1}{c}{ Variáveis } & \multicolumn{1}{c}{ Questões } \\
\hline & Espaço Físico Adequado & $\begin{array}{l}\text { A unidade possui espaço físico suficiente para o } \\
\text { atendimento da população. }\end{array}$ \\
\cline { 2 - 3 } & Equipamentos & A unidade possui equipamentos necessários. \\
\cline { 2 - 3 } & Veículos & $\begin{array}{l}\text { Possui veículos para atendimento. } \\
\text { Ambiente de trabalho adequado }\end{array}$ \\
$\begin{array}{l}\text { Oo trabalho (higiênico ventilado e com os materiais } \\
\text { necessários). }\end{array}$
\end{tabular}

Fonte: Elaboração dos autores, 2013.

A média desse constructo foi de 2,87, maior média dentre os demais, mostrando ser um ponto negativo na visão dos profissionais de saúde. Percebe-se que a variável "espaço físico adequado", média, 3,04, foi considerado um ponto negativo dentro da ESF, podendo prejudicar a efetividade do programa.

Essa realidade também foi encontrada em outros estudos (SANTOS et al., 2008; SILVEIRA; SANTOS; COSTA, 2001) que apontam relatos de problemas de inadequação e manutenção das unidades de saúde e ausência de espaços físicos apropriados para atender a população.

As variáveis "equipamentos" e "veículos" foram as que apresentaram menor média, 2,57, e 2,9 respectivamente. É oportuno salientar que em todas as equipes estudadas, nenhuma relatou possuir veículo próprio para atendimento. Contudo, este era colocado à disposição das equipes em média 2 vezes na semana, mediante agendamento na Secretaria Municipal de Saúde.

As condições de trabalho dos profissionais de saúde são incoerentes com o discurso oficial do Ministério da Saúde. Alguns autores explicam a contradição existente no cenário do SUS, como Borges, Tamayo e Alves Filho (2005) que apontam em seus estudos um paradoxo 

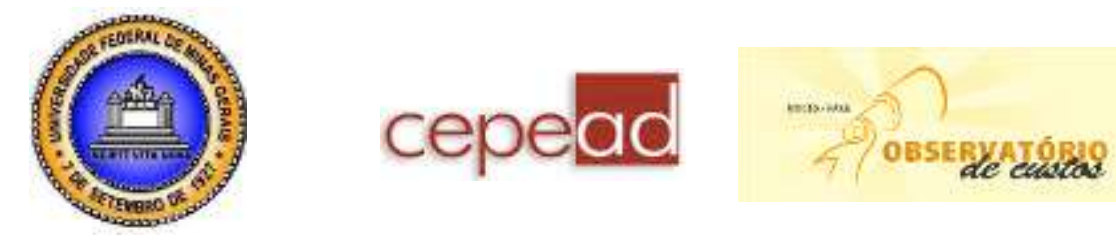

entre os princípios traçados em uma concepção e ideologia centrada no enaltecimento dos propósitos sociais do trabalho enquanto que a realidade vivenciada é marcada por baixos investimentos de recursos.

A falta de investimentos ou a má gestão dos recursos destinados à ESF reflete em unidades sem condição de trabalho adequada, o que compromete a efetividade e eficiência do programa (MARQUES e MENDES, 2005).

\section{Receptividade}

A receptividade esta relacionada à maneira como a equipe de saúde é acolhida pelos usuários do programa.

A Tabela 4 relaciona as variáveis às respectivas questões em que foram fundamentadas.

Tabela 4: Constructo Receptividade

\begin{tabular}{lll}
\hline Constructo & \multicolumn{1}{c}{ Variáveis } & \multicolumn{1}{c}{ Questões } \\
\hline & Demanda & $\begin{array}{l}\text { A população procura a Unidade Básica } \\
\text { quando é necessário. }\end{array}$ \\
\cline { 2 - 4 } & Facilidade de acesso & $\begin{array}{l}\text { A população percebeu uma maior facilidade } \\
\text { ao acesso aos serviços de saúde. }\end{array}$ \\
\cline { 2 - 4 } & Reconhecimento da população & $\begin{array}{l}\text { A população reconhece o trabalho do } \\
\text { Programa. }\end{array}$ \\
\cline { 2 - 4 } & Promoção à saúde & $\begin{array}{l}\text { Ocorrem palestras ou reuniões para } \\
\text { orientação de cuidados com a saúde e } \\
\text { medidas sanitárias. }\end{array}$ \\
\hline
\end{tabular}

Fonte: Elaboração dos autores, 2013.

Esse constructo resultou em uma média igual a 1,98. A variável "demanda" teve maior representatividade na média do constructo, 1,38, o que mostra a procura da população pelas ações de saúde praticadas nas unidades. A literatura aponta relatos de outros autores sobre a incapacidade da ESF de suprir a demanda dos usuários (DIMEINSTEIN; SANTOS, 2005; TRAD et al, 2002 ), mostrando ser uma situação frequente na realidade do sistema público de saúde.

Os resultados revelaram que os profissionais da ESF não se sentem reconhecidos pela população quanto ao trabalho desenvolvido. Essa constatação pode exprimir a não compreensão dos objetivos da ESF por parte da população que atribui ao médico o papel de protagonista dentro do programa.

Os autores Crevelim e Peduzzi (2005) sustentam a importância do reconhecimento da população como componente do processo de construção de um projeto assistencial comum, o que não foi constatado no caso das equipes estudadas onde seus membros não sentem seu trabalho reconhecido pela população. 


\section{Gestão}

Esse constructo apresenta a percepção dos profissionais da Estratégia Saúde da Família quanto ao processo de gestão dentro do programa. As variáveis que o compõe estão apresentadas na tabela abaixo, acompanhadas das respectivas questões em que foram fundamentadas.

A gestão no cenário da Estratégia Saúde da Família constitui-se como um importante instrumento para o êxito das propostas estabelecidas pelo Ministério da Saúde, pois as necessidades de cada UBS são atendidas através de uma boa administração dos recursos utilizados.

$\mathrm{O}$ valor desse constructo foi igual a 2,45. Fica evidente que apesar de ser notória a divisão de funções, esta não é cumprida, sendo comprovada pelo valor apresentado pela variável "competência", que obteve a maior média do total de variáveis consideradas, aproximadamente 3,09. O que se compreende é que o número de profissionais não é suficiente para atender a demanda dos usuários, fazendo com que as funções sejam compartilhadas entre os membros. Essa situação ilustra a necessidade de aperfeiçoamento na gestão dos Recursos Humanos dentro da ESF, de modo que o atendimento possa ser feito de forma integral e contínua.

Tabela 5: Constructo Gestão

\begin{tabular}{|c|c|c|}
\hline Constructo & Variáveis & Questões \\
\hline \multirow{5}{*}{ } & Material & $\begin{array}{l}\text { A realização de pedidos de material não prejudicam } \\
\text { o funcionamento. }\end{array}$ \\
\hline & Documentação & Os processos de documentação são detalhados. \\
\hline & Funções definidas & Existem funções bem definidas. \\
\hline & Competências dos profissionais & $\begin{array}{l}\text { Nenhum funcionário desempenha trabalho além do } \\
\text { que lhe é da sua função. }\end{array}$ \\
\hline & Divisão de funções & É notório a todos da equipe a divisão de funções. \\
\hline
\end{tabular}

Fonte: Elaboração dos autores, 2013.

Essa sobrecarga de trabalho é relatada também por Trad e Rocha (2011) que apontam o cadastramento ultrapassado de usuários, não levando em consideração o número de famílias da área; a pressão da demanda por consultas e exames individuais; a deficiência estrutural das unidades e o reduzido número de auxiliares para o trabalho administrativo como as principais causas para esse excesso. Convém salientar que, apesar de ser notória entre a equipe a divisão de funções, a realidade encontrada evidencia que as tarefas administrativas eram exercidas por um profissional da saúde, devido a limitação de Recursos Humanos.

\section{Carga Horária}

Esse constructo agrupa as variáveis "escala de trabalho", "assiduidade", "controle de frequência" e "punições", apresentadas a seguir: 
Tabela 6: Constructo Carga Horária

\begin{tabular}{|c|c|c|}
\hline Constructo & Variável & Questões \\
\hline \multirow{4}{*}{ 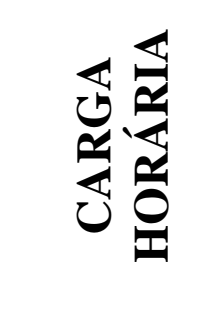 } & Escala de Trabalho & Todos os membros da equipe cumprem sua escala de trabalho. \\
\hline & Assiduidade & São assíduos. \\
\hline & Controle de frequência & Há um controle de frequência de todos os membros. \\
\hline & Punições & $\begin{array}{l}\text { Existem punições em caso de não cumprimento da carga } \\
\text { horária }\end{array}$ \\
\hline
\end{tabular}

Fonte: Elaboração dos autores, 2013.

A média obtida do constructo foi de 2,22 e reflete a opinião dos profissionais quanto a carga horária cumprida por eles. Apesar dos membros concordarem que a carga horária cumprida por eles serem um aspecto positivo, percebe-se pela média da variável "escala de trabalho", 2,85, que nem todos os membros cumprem fielmente a carga horária exigida. Isso porque apesar da existência do controle de frequência, este não é aplicado aos médicos, que não cumprem a totalidade da carga horária proposta pelo Ministério da Saúde, como descrito nos estudos de Araújo e Dimenstein (2006) que relatam essa frequente situação.

\section{Salários}

Os salários representam a remuneração dos profissionais pelos serviços prestados. A Tabela 7 apresenta as variáveis utilizadas nesse constructo.

Tabela 7: Constructo Salários

\begin{tabular}{|c|c|c|}
\hline Constructo & Variáveis & Questões \\
\hline \multirow{3}{*}{ 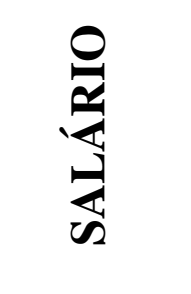 } & Salário coerente com o mercado & Seu salário é coerente como o do mercado. \\
\hline & Salário da equipe & $\begin{array}{l}\text { O salário dos demais membros da equipe também é } \\
\text { coerente com o mercado. }\end{array}$ \\
\hline & Condições de trabalho & São oferecidas condições dignas de trabalho. \\
\hline
\end{tabular}

Fonte: Elaboração dos autores, 2013.

Esse constructo apresentou valor igual a 2,80. Pela percepção dos respondentes, constatou-se que os salários dos membros da equipe são coerentes com o praticado no mercado, obtendo essa variável a melhor média, 2,67.

A variável "condições de trabalho" apresentou uma média de 3,04. Para os entrevistados as condições de trabalho as quais estão submetidas não são dignas para o bom funcionamento das funções que lhes competem. Resultado semelhante foi apontado por outros autores da área que destacam a ausência de espaços físicos apropriados nas unidades 

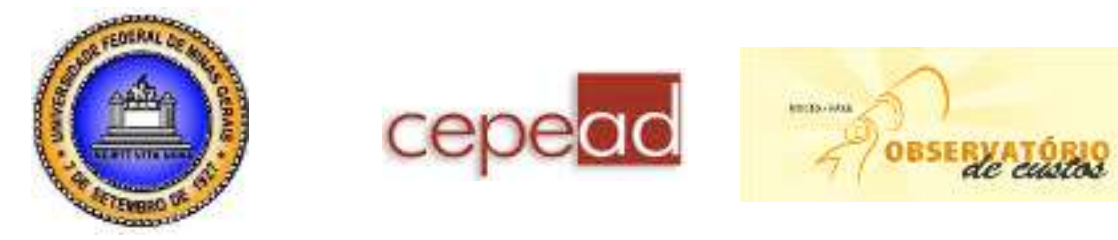

(problemas com tamanho e/ou disposição das salas, climatização, ruídos etc.) como responsáveis pelos efeitos negativos na realização das atividades essenciais do programa (TRAD; ROCHA, 2011).

\section{Resultado}

Neste constructo consideraram-se as variáveis "marcação de consultas", "efeitos secundários" e "resultados amplos". As questões que resultaram nas variáveis estudadas estão apresentadas a seguir:

Tabela 8: Constructo Resultado

\begin{tabular}{cll}
\hline Constructo & \multicolumn{1}{c}{ Variáveis } & \multicolumn{1}{c}{ Questões } \\
\hline \multirow{2}{*}{ Marcação de consultas } & $\begin{array}{l}\text { A marcação de consultas e o tempo de espera atendem de } \\
\text { forma satisfatória à comunidade. }\end{array}$ \\
\cline { 2 - 3 } & Efeitos Secundários & $\begin{array}{l}\text { Você percebe efeitos secundários causados pelas ações do } \\
\text { programa. }\end{array}$ \\
\cline { 2 - 4 } & Resultados amplos & $\begin{array}{l}\text { A partir dos efeitos das ações, existem resultados mais amplos, } \\
\text { influenciados também por outros fatores. }\end{array}$ \\
\hline
\end{tabular}

Fonte: Elaboração dos autores, 2013.

Esse constructo apresenta a visão dos profissionais em relação aos resultados alcançados com as ações desenvolvidas na comunidade em que atuam. Resultou em um valor de 2,19, representando a média das variáveis que o constitui. Os indicadores revelaram que a variável "efeitos secundários", média 2,04, foi a que apresentou a melhor perspectiva positiva dos colaboradores no que tange aos efeitos secundários causados pelas ações do programa.

Observou-se que todas as variáveis analisadas não apresentaram discrepância entre as médias, o que leva a entender que os profissionais percebem um bom resultado alcançado com as ações desenvolvidas. A respeito disso, Krug et al (2010) pontua que trabalhar com uma população adstrita, podendo conhecer as famílias e ter uma noção dos aspectos sociais dos pacientes; fazer um plano de tratamento e ter tempo para um atendimento de qualidade, torna possível ao profissional a visualização dos resultados do trabalho, o que foi sustentado com os resultados da pesquisa.

\section{CONCLUSÃO}

Os apontamentos encontrados na literatura sobre o processo de trabalho dos profissionais foram parcialmente confirmados na pesquisa. Identificou-se que na prática a realidade das equipes de saúde não condiz com as orientações do Ministério da Saúde a cerca do funcionamento do programa.

A observação da realidade das equipes por meio do desenvolvimento do estudo permitiu conhecer as principais limitações da ESF na visão dos profissionais envolvidos. As condições de trabalho, sejam elas materiais ou estruturais, comprometem a qualidade dos serviços de saúde oferecidos nas unidades, refletindo negativamente na satisfação e aceitação da população do novo modelo assistencial. 

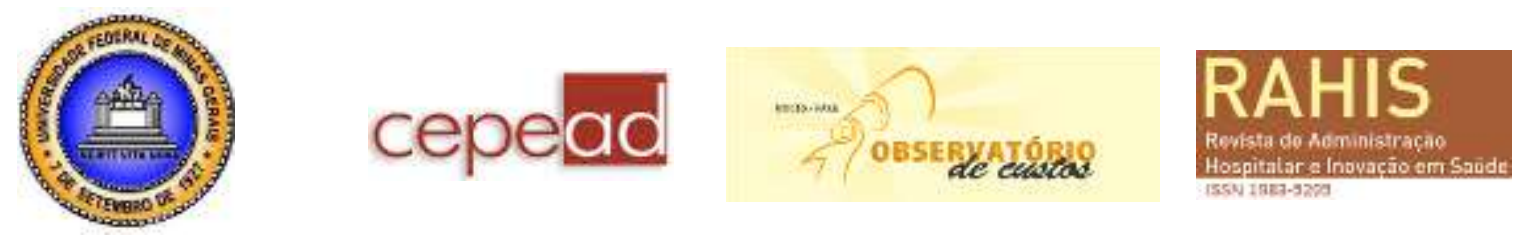

Contudo, percebe-se que as condições de trabalho impostas às equipes nas UBS's em que atuam não constituem um empecilho para a consolidação da ESF como novo modelo assistencial proposto pelo Ministério da Saúde, configurando-se como um de muitos desafios a serem superados. A eficiência da ESF depende da articulação entre os gestores públicos de planejar, organizar e orientar as ações de saúde a serem executadas, destinando recursos suficientes que garantam a qualidade na assistência oferecida à população.

Diante das constatações apresentadas, conclui-se que a ESF atende um dos seus objetivos que é reafirmar os princípios básicos do SUS. A integralidade está presente ao desenvolver as ações de promoção, prevenção e recuperação; atende ao principio da universalidade através do acesso gratuito aos serviços de saúde; favorece a participação popular por meio do vínculo criado entre as equipes de saúde e os usuários do sistema o que permite aos mesmos participarem do processo organizacional da ESF e a equidade ao atender as necessidades especificas de cada cidadão.

Espera-se que os achados desta pesquisa possam contribuir nos debates a cerca da efetividade e eficiência dos serviços de saúde na Estratégia Saúde da Família. Conhecer o processo de trabalho dos agentes facilita na identificação dos problemas existentes e permite, ao comparar a realidade com as propostas estabelecidas, aperfeiçoar a operacionalização do sistema, corrigindo as falhas identificadas priorizando sempre a eficiência na Atenção Primária.

\section{REFERÊNCIAS BIBLIOGRÁFICAS}

ALVES e SILVA, A. C. M. et al. A Estratégia Saúde da Família: motivação, preparo e trabalho segundo médicos que atuam em três distritos do município de Duque de Caxias, Rio de Janeiro, Brasil. Revista Saúde e Sociedade, São Paulo, v. 19, n. 1, p. 159$169,2010$.

AZEREDO, C. M. et al. Avaliação das condições de habitação e saneamento: a importância da visita domiciliar no contexto do Programa de Saúde da Família. Revista Ciência \& Saúde Coletiva, Rio de Janeiro, v. 12, n. 3, p. 743-753, mai/jun. 2007.

AYRES, R. C. V. et al. Acolhimento no PSF: humanização e solidariedade. Revista O Mundo da Saúde, São Paulo, v. 30, n. 2, p. 306-311, abr/jun. 2006.

BAZON, F. V. M.; CAMPANELLI, E. A.; BLASCOVI-ASSIS, S. M. A importância da humanização profissional no diagnóstico das deficiências. Revista Psicol. Teor. Prat, São Paulo, v. 6, n. 2, p. 89-99, dez. 2004.

BRASIL. Constituição, 1988. Constituição da República Federativa do Brasil: Constituição do Brasil, promulgada em 5 de outubro de 1988. Brasília (DF): Senado Federal; 1988. Disponível em: <http://www.planalto.gov.br/ccivil_03/constituicao/constituicao.htm>. Acesso em: 20 de Novembro de 2012. 

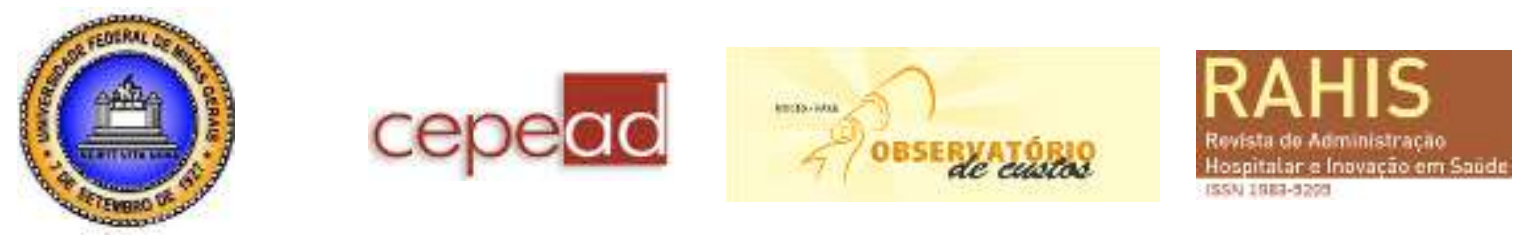

BRASIL, Departamento de Atenção Básica. Atenção Básica e Saúde da Família - Diretriz Conceitual. Disponível em: $\langle$ http://dab.saude.gov.br/atencaobasica.php $>$. Acesso em 15 de Fevereiro de 2013a.

BRASIL, Portal da Saúde. Sistema Único de Saúde. Disponível em: $<$ http://portal.saude.gov.br/portal/saude/visualizar_texto.cfm?idtxt=24627>. Acesso em $20 \mathrm{de}$ Fevereiro de 2013b.

BRASIL, Ministério da Saúde. Secretaria da Assistência à Saúde. Coordenação de Saúde da Comunidade. Saúde da Família: uma estratégia para a reorientação do modelo assistencial. Brasília: Ministério da Saúde, 1997.

CAMELO, S. H. H.; ANGERAMI, E. L. S. Formação de recursos humanos para a estratégia de saúde da família. Revista Ciência, Cuidado e Saúde, Maringá, v. 7, n. 1, p. 4552, jan/mar. 2008.

CARVALHO, S. R.; CUNHA, G. T. A. Gestão da Atenção na Saúde: Elementos para se pensar a mudança da organização na saúde. In: CAMPOS, G.W.S (org). Tratado de Saúde Coletiva. São Paulo: Hucitec, p. 837-868, 2006.

COSTA, F. B; TRINDADE, M. A.N.; PEREIRA, M. L. T. A Inserção do Biomédico no Programa de Saúde da Família. Revista Eletrônica Novo Enfoque, v. 11, n. 11, p. 27- 33, 2010.

COTTA, R. M. et al. Organização do trabalho e perfil dos profissionais do Programa Saúde da Família: um desafio na reestruturação da atenção básica em saúde. Epidemiologia e Serviços de Saúde, Brasília, v.15, n.3, p. 7-18, set. 2006.

CREVELIM, M. A.; PEDUZZI, M. A participação da comunidade na equipe de saúde da família. Como estabelecer um projeto comum entre trabalhadores e usuários? Revista Ciência \& Saúde Coletiva, Rio de Janeiro, v. 10, n. 2, p. 323-331, abr. 2005.

DALPIAZ, A. K.; STEDILE, N. L. Estratégia Saúde da Família: reflexão sobre algumas de suas premissas. In: Jornada Internacional de Políticas Públicas- Estado, Desenvolvimento e Crise do Capital, 5, 2011, Maranhão. Anais...Maranhão: Jornada Internacional de Políticas Públicas- UFMA, 2011.

GIACOMOZZI, C. M.; LACERDA, M. R.A Prática da assistência domiciliar dos profissionais da Estratégia de Saúde da Família. Texto \& Contexto- Enfermagem, Florianópolis, v. 15, n. 4, p. 645-653, 2006.

GIL, A. C. Métodos e Técnicas de Pesquisa Social. 5ª Ed. São Paulo: Atlás, 2006.

HAIR, Jr., Joseph F.; ANDERSON, Rolph E.; TATHAM, Ronald L. e BLACK, William C. Análise Multivariada de Dados. $6^{\text {a }}$ edição, Porto Alegre: Bookman, 2009. 

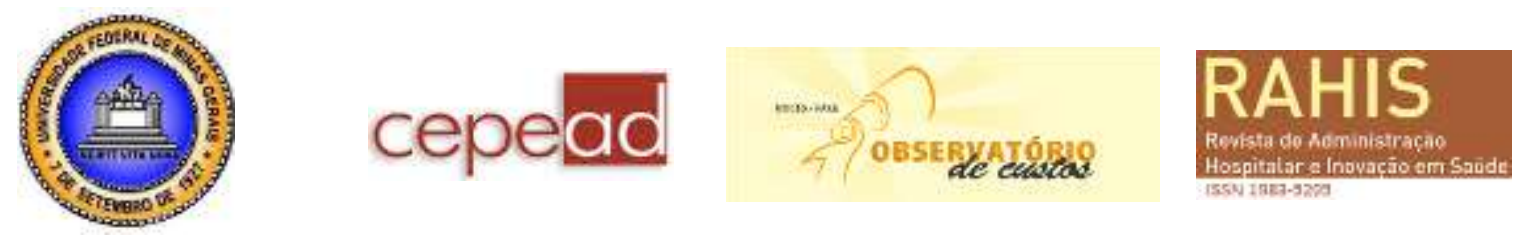

L'ABBATE, S. Educação em saúde: uma nova abordagem. Cadernos Saúde Pública, Rio de Janeiro, v. 10, n. 4, dez. 1994.

MAIA, M. A. C.; SANTOS, J. S. Qualidade de vida no Programa Saúde da Família: A visão de agentes comunitários de saúde e usuários. Revista Brasileira Promoção da Saúde. Unifor, Fortaleza, v. 24, n. 3, p. 228-237, 2011.

MALHOTRA, N. K. Pesquisa de Marketing: uma orientação aplicada.4 $4^{\text {a }}$ Ed. Porto Alegre: Bookman, 2006.

MARTA, S. N. et al. Programa Saúde da Família sob a visão do usuário. Salusvita, Bauru, v. 30, n. 3, p. 159-177, 2011.

MARQUI, A. B. T. et al . Caracterização das equipes da Saúde da Família e de seu processo de trabalho. Revista Escola de Enfermagem USP, São Paulo, v. 44, n. 4, p. 956961, dez.. 2010.

ROSA, W. A. G.; LABATE, R. C. Programa saúde da família: a construção de um novo modelo de assistência. Revista Latino-Americana de Enfermagem, Ribeirão Preto, v. 13, n. 6, dez. 2005.

RIBEIRO, E. M.; PIRES, D.; BLANK, V. L. G. A teorização sobre processo de trabalho em saúde como instrumental para análise do trabalho no Programa Saúde da Família. Caderno Saúde Pública, Rio de Janeiro, v. 20, n. 2, p. 438-446, abr. 2004.

SANCHEZ, H. F; DRUMOND, M. M; VILAÇA, E. L. Adequação de recursos humanos ao PSF: percepção de formandos de dois modelos de formação acadêmica em odontologia. Revista Ciência \& Saúde Coletiva, Rio de Janeiro, v. 13, n. 2, p.523-531, mar/ abr. 2008.

SANTOS, S. M. R; JESUS, M.C.P; AMARAL, A.M.M; COSTA, D.M.N; ARCANJO, R. A. A consulta de enfermagem no contexto da atenção básica de saúde. Texto \& contexto Enfermagem, Juiz de Fora, Minas Gerais, 2008; v. 17, n. 1, p.124-130, 2008.

SILVEIRA, D. S; SANTOS, I. S; COSTA, J.S.D. Atenção pré-natal na rede básica: uma avaliação da estrutura e do processo. Caderno Saúde Publica, v. 17, n. 1, p. 131-139, 2001.

TRAD, L. A. B.; ROCHA, A. A. R. M. Condições e processo de trabalho no cotidiano do Programa Saúde da Família: coerência com princípios da humanização em saúde. Revista Ciência \& Saúde Coletiva, Rio de Janeiro, v. 16, n. 3,p. 1969- 1980, Mar. 2011.

VIANA, A. L.; DAL POZ, M. R.A Reforma do Sistema de Saúde no Brasil e o Programa de Saúde da Família. Physis, Rio de Janeiro, v. 8, n. 2, p. 11-48, Jul/Dez. 1998. 